\title{
Comparison of Surgical Resection and Systemic Treatment for Hepatocellular Carcinoma with Vascular Invasion: National Cancer Database Analysis
}

\author{
Rajalakshmi Govalan ${ }^{a}$ Marie Lauzon ${ }^{b, c}$ Michael Luu ${ }^{b, c}$ Joseph C. Ahn ${ }^{d}$ \\ Kambiz Kosarib,e,f Tsuyoshi Todob, e,f Irene K. Kim ${ }^{e, f}$ Mazen Noureddin ${ }^{a, e, g}$ \\ Alexander Kuo $^{\mathrm{a}, \mathrm{e}, \mathrm{g}}$ Ayoub S. Walida, e,g Vinay Sundaramª, ${ }^{\mathrm{a}}$ Shelly C. Lu ${ }^{\mathrm{a}, \mathrm{b}}$ \\ Lewis R. Roberts ${ }^{d}$ Amit G. Singal ${ }^{\text {h, i }}$ Julie K. Heimbach ${ }^{\mathrm{j}, \mathrm{k}}$ Vatche G. Agopian \\ Nicholas Nissen ${ }^{b, e, f}$ Ju Dong Yang ${ }^{a, b, e, g}$

\begin{abstract}
aDepartment of Medicine, Cedars-Sinai Medical Center, Los Angeles, CA, USA; bSamuel Oschin Comprehensive Cancer Institute, Cedars-Sinai Medical Center, Los Angeles, CA, USA; 'Biostatistics and Bioinformatics Research Center, Cedars-Sinai Medical Center, Los Angeles, CA, USA; ${ }^{\mathrm{d} D}$ Division of Gastroenterology and Hepatology, Mayo Clinic, Rochester, MN, USA; ${ }^{e}$ Comprehensive Transplant Center, Cedars-Sinai Medical Center, Los Angeles, CA, USA; fDepartment of Surgery, Cedars-Sinai Medical Center, Los Angeles, CA, USA; ${ }^{9}$ Karsh Division of Gastroenterology and Hepatology, Cedars-Sinai Medical Center, Los Angeles, CA, USA; ${ }^{h}$ Harold C. Simmons Comprehensive Cancer Center, UT Southwestern Medical Center, Dallas, TX, USA; 'Division of Digestive and Liver Diseases, UT Southwestern

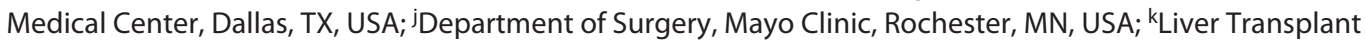
Program, Mayo Clinic, Rochester, MN, USA; 'Department of Surgery, David Geffen School of Medicine at UCLA, Los Angeles, CA, USA
\end{abstract}

\section{Keywords}

Hepatocellular carcinoma · Vascular invasion · Surgical resection · Prognosis

\begin{abstract}
Introduction: Small studies from outside of the USA suggest excellent outcomes after surgical resection for hepatocellular carcinoma (HCC) with vascular invasion. The study aims to (1) compare overall survival after surgical resection and systemic therapy among patients with HCC and vascular invasion and (2) determine factors associated with receipt of surgical resection in a US population. Methods: $\mathrm{HCC}$ patients with AJCC clinical TNM stage 7th T3BNOMO diagnosed be-
\end{abstract}

karger@karger.com www.karger.com/lic

Karger $\stackrel{\text { ' }}{5}$

GOPEN ACCESS
(C) 2021 The Author(s)

Published by S. Karger AG, Basel

This is an Open Access article licensed under the Creative Commons Attribution-NonCommercial-4.0 International License (CC BY-NC) (http://www.karger.com/Services/OpenAccessLicense), applicable to the online version of the article only. Usage and distribution for commercial purposes requires written permission. tween 2010 and 2017 from the National Cancer Database were analyzed. Cox and logistic regression analyses identified factors associated with overall survival and receipt of surgical resection. Results: Of 11,259 patients with T3BNOM0 HCC, 325 (2.9\%) and 4,268 (37.9\%) received surgical resection and systemic therapy, respectively. In multivariable analysis, surgical resection was associated with improved survival compared to systemic therapy (adjusted hazard ratio: $0.496,95 \%$ confidence interval: $0.426-0.578$ ) with a median survival of 21.4 and 8.1 months, respectively. Superiority of surgical resection was observed in noncirrhotic and cirrhotic subgroups and propensity score matching and inverse probability of treatment weighting adjusted analysis. Asians were more likely to receive surgical resection, whereas Charl- 
son comorbidity $\geq 3$, elevated alpha-fetoprotein, smaller tumor size, care in a community cancer program, and the South or West region were associated with a lower likelihood of surgical resection. Conclusion: HCC patients with vascular invasion may benefit from surgical resection compared to systemic therapies. Demographic and clinical features of HCC patients and region and type of treating facility were associated with surgical resection versus systemic treatment.

(C) 2021 The Author(s)

Published by S. Karger AG, Basel

\section{Introduction}

Hepatocellular carcinoma (HCC) is the fourth leading cause of cancer-related mortality worldwide, and the rates continue to rise $[1,2]$. Unlike most other solid cancers, prognosis and management of HCC depend not only on the tumor burden (size, vascular invasion, and extrahepatic spread) but also on other factors including patient's performance status and degree of liver dysfunction [3]. A number of integrated staging classification systems incorporating these factors have been developed to predict prognosis [4]. Of these, the Barcelona Clinic Liver Cancer (BCLC) classification system and, more recently, the Hong Kong Liver Cancer staging system are used to predict prognosis and pair suggested treatment algorithms to different stages [5-8].

In spite of advancements in routine surveillance efforts to detect HCC at an early stage, the majority of patients are being diagnosed at an advanced stage with limited treatment options leading to poor outcomes $[9,10]$. HCC patients with macrovascular (portal or hepatic vein) invasion are categorized as advanced-stage HCC (BCLC stage $\mathrm{C}$ ); however, this category is heterogeneous and includes HCC patients with vascular invasion, extrahepatic spread, and/or those with cancer-related symptoms [7]. Until recently, the only approved first-line agent for advanced HCC was sorafenib, a multikinase inhibitor that has shown to improve overall survival compared to placebo $[11,12]$. However, sorafenib only has marginal survival benefits for those with vascular invasion $[13,14]$.

Surgical resection is one of the curative treatment options for HCC and associated with 5-year survival exceeding $60 \%$ but is typically restricted to patients with earlystage HCC $[8,14]$, so only a minority of patients with HCC are eligible for surgical resection $[9,15]$. In many US centers, vascular invasion, regardless of the degree of invasion, is considered a contraindication to surgery [7, 8], although this has been challenged by data among hep- atitis B-infected patients from Asian centers, suggesting that some patients with vascular invasion might benefit from resection [16-20]. These data require validation in Western patient populations, given differences in liver disease etiologies and comorbidities, but there are only a few small-scale studies from the USA to date $[18,20]$. The aims of this study were to (1) compare overall survival after surgical resection and systemic therapy in a US cohort of patients with HCC and vascular invasion and (2) identify factors associated with the receipt of surgical resection.

\section{Method}

\section{Database}

The National Cancer Database (NCDB) is a joint project of the Commission on Cancer $(\mathrm{CoC})$ of the American College of Surgeons and the American Cancer Society. It is a clinical oncology database sourced from hospital registry data that are collected in more than 1,500 CoC-accredited facilities. Data represent more than 70 percent of newly diagnosed cancer cases nationwide and more than 34 million historical records. The study is exempt from Ethical Committee approval as a de-identified dataset from the NCDB was analyzed in the current study.

\section{Patients and Variables}

All HCC patients with AJCC 7th clinical stage T3BNOM0 (tumor of any size involving a major branch of the portal vein or hepatic vein without lymph node or extrahepatic metastasis) who were diagnosed between 2010 and 2017 were identified from the NCDB. HCC diagnosis was based on the International Classification of Disease-Oncology-3rd Edition code C22.0 and the histology codes $8170-8175$. Patients with missing treatment information and those who did not receive surgical resection or systemic treatment were excluded.

Surgical resection was defined using the variable "Surgical Procedure of Primary Site" which records the surgical or ablation procedure performed at the primary site at any facility after excluding patients who underwent liver transplantation or ablation. Systemic treatment was defined using variables "Chemotherapy" and "Immunotherapy" which record the type of chemotherapy or immunotherapy administered as the first-course treatment at any facility. Patients who received both surgical resection and systemic treatment were classified under surgery considering the definitive nature of the surgical treatment.

Demographic, socioeconomic, reporting medical facilities, and clinical information of patients were extracted from the NCDB. Demographic data included age, sex, and race/ethnicity. Socioeconomic data included insurance status, income (median household income for patient zip code based on 2016 American Community Survey data, in quartiles), education (median percentage of adults aged 25 years or older in the patient's zip code without a high school degree based on 2016 American Community Survey data), and urban/rural status (metropolitan, urban, and rural). Facility data included type (academic: $>500$ new cancer diagnoses annually and at least 4 postgraduate training programs; comprehensive community: >500 new cancer diagnoses annually; integrated net- 
Table 1. Clinical features of patients before and after propensity score matching

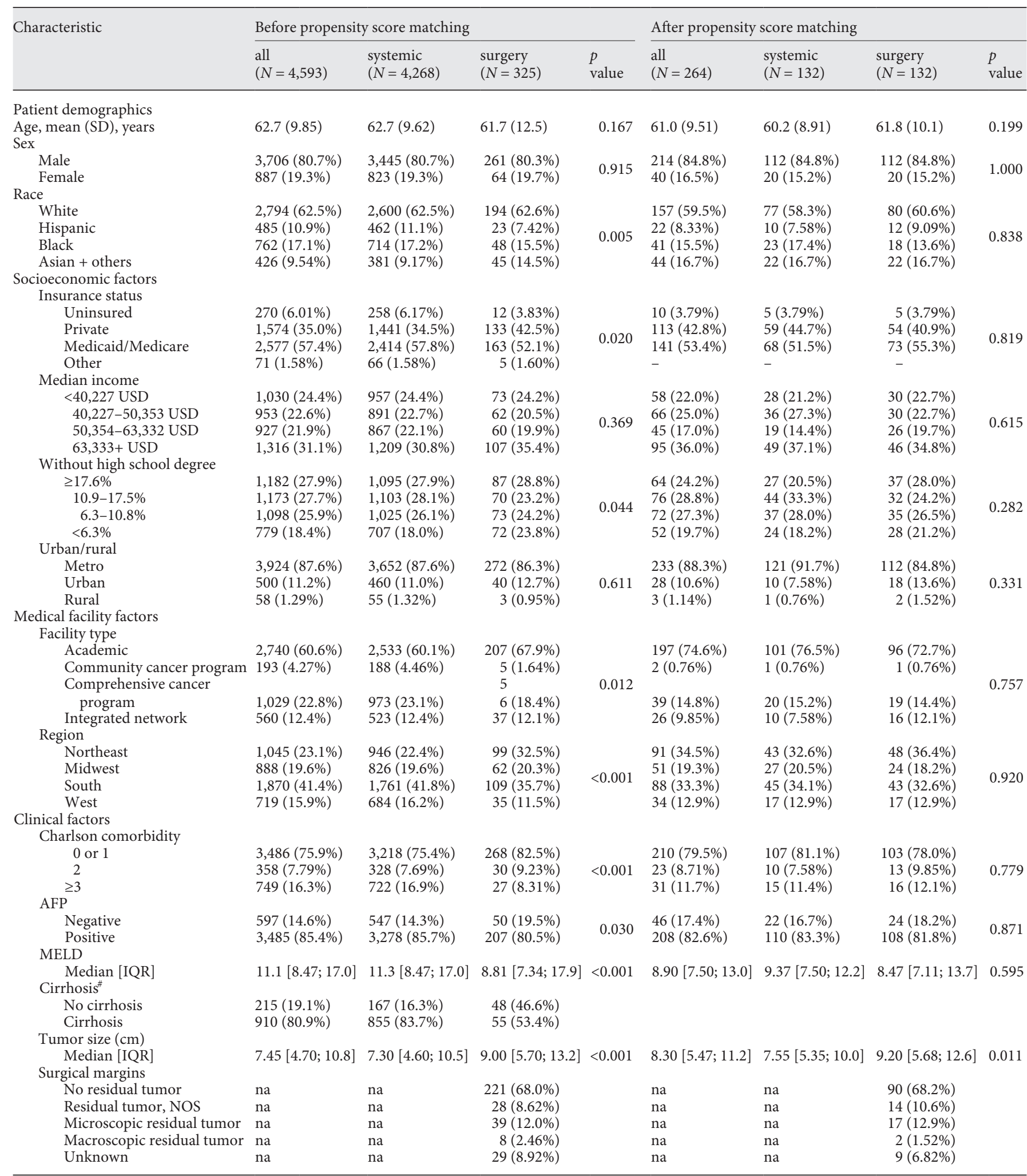

AFP, alpha-fetoprotein; na, not applicable. ${ }^{\#}$ Cirrhosis status is available in only $24 \%$, thus was not considered in propensity score matching in the main analysis, but sensitivity analysis was performed.

Hepatocellular Carcinoma with Vascular Invasion
Liver Cancer 2021:10:407-418

DOI: $10.1159 / 000515554$ 
work: no minimum caseload, a joint venture with multiple facilities providing integrated cancer care with at least one facility being a hospital and a CoC-accredited cancer program; and community: 100-500 new cancer diagnoses annually) and geographic location of the medical center. Clinical data included Charlson/Deyo comorbidity score $(0,1,2$, and $\geq 3$ ), alpha-fetoprotein (AFP) (normal vs. elevated), cirrhosis per Ishak fibrosis stages 5-6, MELD score, treatment, tumor size, and surgical margins.

\section{Statistical Analysis}

Baseline and demographic characteristics were summarized by standard descriptive measures (frequency and percentage for categorical variables and mean [standard deviation] or median [range] for continuous variables). These characteristics were then compared by a treatment group using Pearson's $\chi^{2}$ test for categorical variables and Welch's $t$-test or the Mann-Whitney-Wilcoxon test for continuous variables as appropriate. Survival probabilities were estimated using the Kaplan-Meier method and compared using the log-rank test. Factors associated with overall survival were determined using univariable and multivariable Cox proportional hazards regression. Time-to-event is defined as the time from HCC diagnosis to last follow-up or death. For missing data in the $\mathrm{NCDB}$, the chained equation approach for multiple imputations was used [21].

We also compared overall survival among patients who received surgical resection versus systemic treatment using propensity score-matched analysis, which was matched by all demographic and clinical variables (age, sex, race/ethnicity, insurance, comorbidity, AFP level, MELD score, facility type, geographic region, and tumor size), as well as inverse probability of treatment weighting (IPTW) [22]. Propensity scores were constructed using a multivariable logistic regression model for each patient among each pair of treatments. Propensity score-matched cohorts were constructed by performing a 1:1 match among patients between each of the treatments with a caliper of 0.10 using the nearest neighbor method [23]. Balance diagnostics among each pair of matched treatment cohorts were assessed by the standardized mean differences and visually with the histograms of the propensity score distributions. IPTW was constructed based on the propensity scores and included in the Cox proportional hazard regression model as case weights. The proportional hazards assumption among all survival models was assessed by the scaled Schoenfeld residuals as well as the goodness-of-fit test as proposed by Grambsch and Therneau [24].

Finally, we also identified factors associated with receipt of surgical HCC treatment using univariable and multivariable logistic regression. All statistical analyses were performed using R statistical software (version 4.0.3; R Foundation, Vienna, Austria) with two-sided tests and a significance level of 0.05 .

\section{Results}

\section{Patient Characteristics}

Of 11,259 patients with T3BN0M0 HCC diagnosed between 2010 and 2017, 325 (2.9\%) and 4,268 (37.9\%) patients received surgical resection and systemic therapy, respectively (shown in online suppl. Fig. 1; see www. karger.com/doi/10.1159/000515554 for all online suppl. material). Table 1 shows the demographic, socioeconomic, and clinical characteristics of the patient population. The mean age of the population was 62.7 and the majority were men. The population was racially diverse and evenly distributed in terms of their income and high school education. Approximately $60.6 \%$ were treated at academic centers, followed by comprehensive cancer programs $(22.8 \%)$, integrated network $(12.4 \%)$, and community programs (4.3\%). Most patients $(85.4 \%)$ had increased AFP levels at HCC diagnosis. Cirrhosis status was missing in $76 \%$ of the patients. Among $24 \%$ of patients with cirrhosis status data, the proportion of patients with cirrhosis was much lower in patients who underwent surgical resection than those who received systemic therapy. Lobectomy (43.1\%) was the most common type of surgical resection followed by wedge or segmental resection (33.5\%) and extended lobectomy (17.5\%). Surgery type was not specified in $5.8 \%$ of resected patients. The population characteristics were comparable for most variables after propensity score matching except for the tumor size: size was larger in surgical resection than systemic treatment group (Table 1).

\section{Prognostic Factors in T3BNOMO HCC}

Cox proportional hazards analyses showed that female sex (adjusted hazard ratio [AHR]: 0.873, 95\% CI: $0.793-$ 0.962 ) and Hispanic ethnicity (AHR: $0.866,95 \%$ CI: $0.752-0.997)$ were associated with improved overall survival (OS) compared to male sex and white race, respectively (Table 2). Patients receiving HCC treatment at community cancer centers (AHR: 1.299, 95\% CI: 1.0651.585), comprehensive community cancer programs (AHR: 1.174, 95\% CI: 1.065-1.294), and integrated network program (AHR: 1.145, 95\% CI: 1.017-1.288) had decreased OS compared to patients receiving treatment at academic medical centers. Additionally, elevated AFP (AHR: 1.278, 95\% CI: 1.132-1.443) and higher MELD scores (AHR per 10 units: 1.137, 95\% CI: 1.084-1.193) were significantly associated with shorter OS.

\section{Impact of Surgical Resection on Overall Survival}

Rates for rehospitalization and mortality within 30 days after surgical resection were 10.2 and $8.5 \%$, respectively. The median survival for patients treated with surgical resection was 21.4 months compared to 8.1 months for those treated with systemic therapy as a first-line treatment (shown in Fig. 1). In Cox proportional hazards analysis, patients who received surgical resection (AHR: $0.496,95 \%$ CI: $0.426-0.578$ ) had a $50 \%$ reduction in risk 
Table 2. Prognostic factors among patients who received surgical resection or systemic treatment for HCC with vascular invasion

\begin{tabular}{|c|c|c|c|c|}
\hline Characteristic & Univariable HR (95\% CI) & $p$ value & Multivariable HR (95\% CI) & $p$ value \\
\hline \multicolumn{5}{|l|}{ Patient demographics } \\
\hline Age (10-year change) & $1.025(0.990-1.061)$ & 0.162 & $1.018(0.977-1.061)$ & 0.395 \\
\hline \multicolumn{5}{|l|}{ Sex } \\
\hline Male (reference) & (Reference) & & (Reference) & \\
\hline Female & $0.884(0.810-0.965)$ & 0.006 & $0.873(0.793-0.962)$ & 0.006 \\
\hline \multicolumn{5}{|l|}{ Race } \\
\hline Hispanic & $0.812(0.721-0.913)$ & $<0.001$ & $0.866(0.752-0.997)$ & 0.045 \\
\hline Black & $0.938(0.854-1.032)$ & 0.188 & $0.939(0.837-1.052)$ & 0.276 \\
\hline Asian + others & $0.858(0.760-0.970)$ & 0.014 & $0.886(0.773-1.015)$ & 0.081 \\
\hline \multicolumn{5}{|l|}{ Socioeconomic factors } \\
\hline \multicolumn{5}{|l|}{ Insurance status } \\
\hline Uninsured (reference) & (Reference) & & (Reference) & \\
\hline \multicolumn{5}{|l|}{ Median income } \\
\hline$<40,227$ USD (reference) & (Reference) & & (Reference) & \\
\hline 40,227-50,353 USD & $1.008(0.903-1.126)$ & 0.887 & $0.984(0.867-1.116)$ & 0.802 \\
\hline $50,354-63,332$ USD & $0.944(0.847-1.052)$ & 0.301 & $0.878(0.759-1.017)$ & 0.083 \\
\hline $63,333+$ USD & $0.976(0.886-1.074)$ & 0.616 & $0.950(0.815-1.107)$ & 0.514 \\
\hline \multicolumn{5}{|l|}{ Without high school degree } \\
\hline$\geq 17.6 \%$ (reference) & (Reference) & & (Reference) & \\
\hline $10.9-17.5 \%$ & $1.096(0.995-1.208)$ & 0.064 & $1.080(0.958-1.218)$ & 0.207 \\
\hline $6.3-10.8 \%$ & $1.145(1.041-1.261)$ & 0.006 & $1.152(1.010-1.315)$ & 0.035 \\
\hline$<6.3 \%$ & $1.122(1.003-1.254)$ & 0.044 & $1.136(0.959-1.347)$ & 0.141 \\
\hline \multicolumn{5}{|l|}{ Urban/rural } \\
\hline Metro (reference) & (Reference) & & (Reference) & \\
\hline Urban & $1.104(0.989-1.231)$ & 0.077 & $1.047(0.919-1.193)$ & 0.488 \\
\hline Northeast (reference) & (Reference) & & (Reference) & \\
\hline Midwest & $1.231(1.107-1.369)$ & $<0.001$ & $1.082(0.960-1.221)$ & 0.197 \\
\hline South & $1.061(0.969-1.161)$ & 0.202 & $0.933(0.840-1.037)$ & 0.198 \\
\hline West & $1.078(0.962-1.208)$ & 0.195 & $0.986(0.867-1.122)$ & 0.834 \\
\hline \multicolumn{5}{|l|}{ Clinical factors } \\
\hline \multicolumn{5}{|l|}{ Charlson comorbidity } \\
\hline 0 or 1 (reference) & (Reference) & & (Reference) & \\
\hline 2 & $0.982(0.858-1.122)$ & 0.786 & $0.925(0.796-1.076)$ & 0.313 \\
\hline 3 & $1.132(1.030-1.245)$ & 0.010 & $1.140(1.025-1.267)$ & 0.016 \\
\hline \multicolumn{5}{|l|}{ AFP } \\
\hline Normal (reference) & (Reference) & & (Reference) & \\
\hline Elevated & $1.247(1.118-1.391)$ & $<0.001$ & $1.278(1.132-1.443)$ & $<0.001$ \\
\hline MELD (10-unit change) & $1.148(1.101-1.198)$ & $<0.001$ & $1.137(1.084-1.193)$ & $<0.001$ \\
\hline Tumor size (1-cm change) & $1.010(1.006-1.014)$ & $<0.001$ & $1.011(1.007-1.015)$ & $<0.001$ \\
\hline \multicolumn{5}{|l|}{ Treatment } \\
\hline Systemic treatment (reference) & (Reference) & & (Reference) & \\
\hline Surgical treatment & $0.472(0.408-0.546)$ & $<0.001$ & $0.496(0.426-0.578)$ & $<0.001$ \\
\hline
\end{tabular}




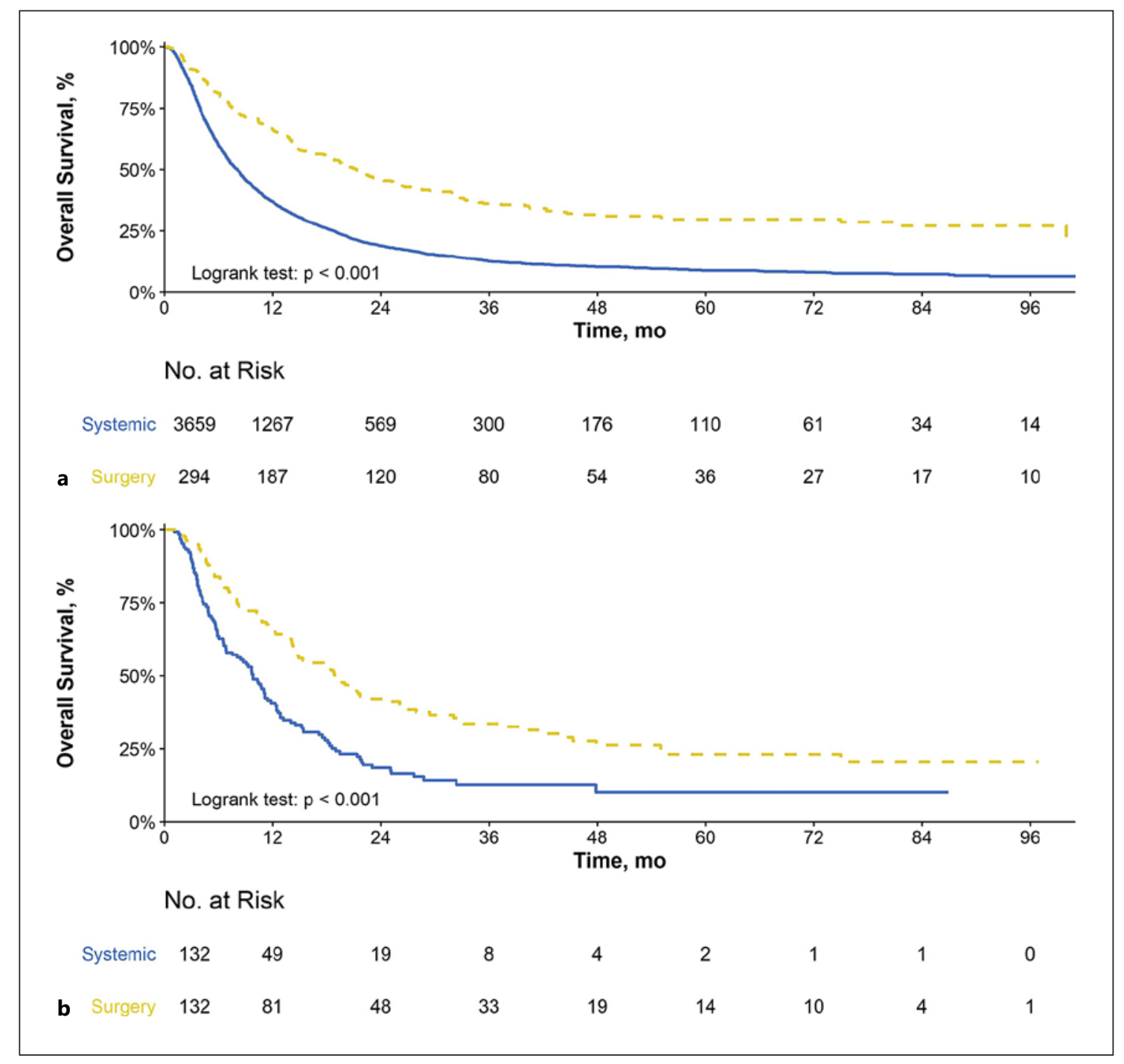

Fig. 1. Kaplan-Meier survival analysis of patients with surgical resection vs. systemic therapy for their HCC with vascular invasion. a Before propensity score matching. b After propensity score matching. HCC, hepatocellular carcinoma.

of mortality compared to those who received systemic therapy. As patients with HCC and major vascular invasion often have elevated bilirubin levels despite preserved liver function due to the intravascular hemolysis, arterioportal shunt, and portal biliopathy, we repeated the analysis with individual components of MELD rather than MELD. The result remains the same (AHR: 0.492, 95\% CI: 0.408-0.594). Finally, consistent results were observed in survival analyses after propensity score matching (AHR: 0.423, 95\% CI: 0.307-0.583) and IPTW-adjusted analysis (AHR: 0.576, 95\% CI: 0.467-0.709).

Despite the adjustment of MELD score, patients who underwent surgery were less likely to have cirrhosis.
Therefore, a sensitivity analysis was conducted to compare OS in a subgroup of patients with/without cirrhosis. Surgical resection was associated with the prolonged OS than systemic treatment in both noncirrhotic (AHR: 0.550, 95\% CI: 0.326-0.926) and cirrhotic (AHR: 0.460, 95\% CI: 0.311-0.679) HCC patients (shown in online suppl. Fig. 2, 3). Finally, we repeated the analysis after propensity score matching of covariates including cirrhosis status with 48 patients in each group and found that surgical resection was associated with the prolonged OS than systemic treatment (AHR: 0.325, 95\% CI: 0.1860.568 ) (shown in online suppl. Fig. 4). 


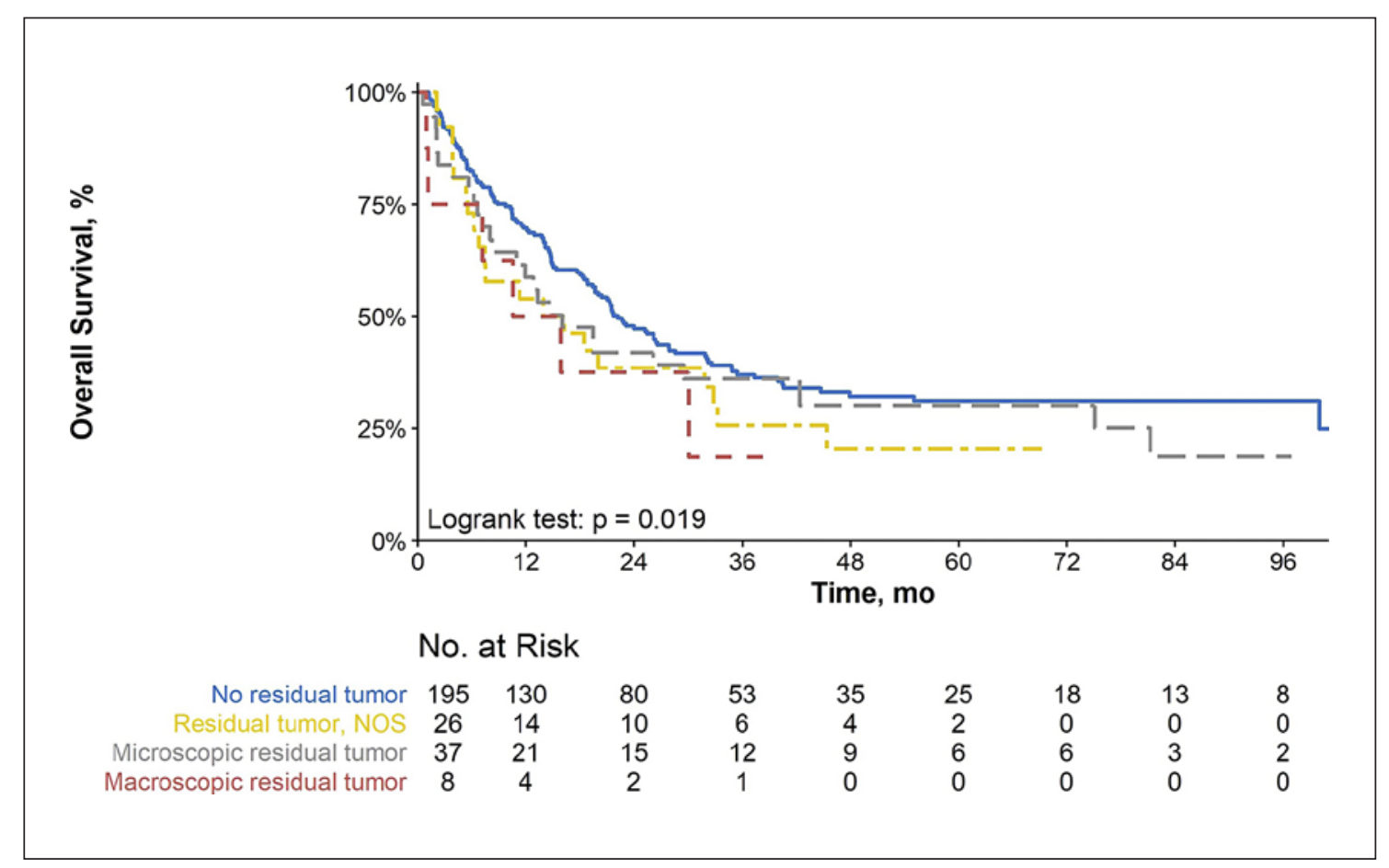

Fig. 2. Survival estimate for HCC patients with vascular invasion who underwent surgical resection by surgical margin status. HCC, hepatocellular carcinoma.

Impact of Surgical Margin on Overall Survival

As expected, residual disease after resection influenced the overall survival of patients treated with surgical resection (shown in Fig. 2). Patients who had complete resection with no residual disease (R0: all margins are grossly and microscopically negative) had a median survival of 22.2 months compared to 16.0 months for those who had microscopic (R1: margins are involved by microscopic residual tumor but cannot be seen by the naked eye) and 13.2 for macroscopic residual disease (R2: gross tumor of the primary site which is visible to the naked eye) ( $p=$ 0.019). Of note, patients who had R1 (AHR: $0.558,95 \%$ CI 0.381-0.819) still had improved survival compared to patients who received systemic treatment as a first-line treatment for HCC.

\section{Factors Associated with HCC Surgical Resection}

versus Systemic Treatment

In univariable and multivariable analyses, patient demographics, medical facility type, region, and patient clinical factors were significantly associated with an increased likelihood of receiving surgical resection (Table 3). Asians (adjusted odds ratio [AOR]: 1.490, 95\% CI: 1.040-2.135) were more likely to receive surgical resec- tion than whites. Patients receiving care at community cancer programs (AOR: 0.431, 95\% CI: 0.187-0.995) were less likely to receive surgical resection than those receiving care at academic centers. In addition, patients treated at centers located in the South (AOR: 0.616, 95\% CI: 0.458-0.829) and the West (AOR: 0.461, 95\% CI: 0.3060.695 ) were less likely to have surgical intervention for their HCC than those treated in the Northeast. As expected, having a Charlson comorbidity score of 3 or higher decreased the likelihood of getting surgical resection (AOR: 0.443, 95\% CI: 0.292-0.672) compared to Charlson comorbidity of 0 or 1 . Finally, elevated AFP level was inversely associated with resection (AOR: 0.697, 95\% CI: $0.512-0.949)$, while a larger size of the tumor was associated with surgical resection (AOR: 1.017, 95\% CI: 1.0061.029).

\section{Discussion}

Our analysis of the NCDB on a contemporary cohort of HCC patients with vascular invasion demonstrated that surgical resection offered a survival benefit compared to systemic therapy, the current standard of care. 
Table 3. Factors associated with surgical resection for HCC with vascular invasion

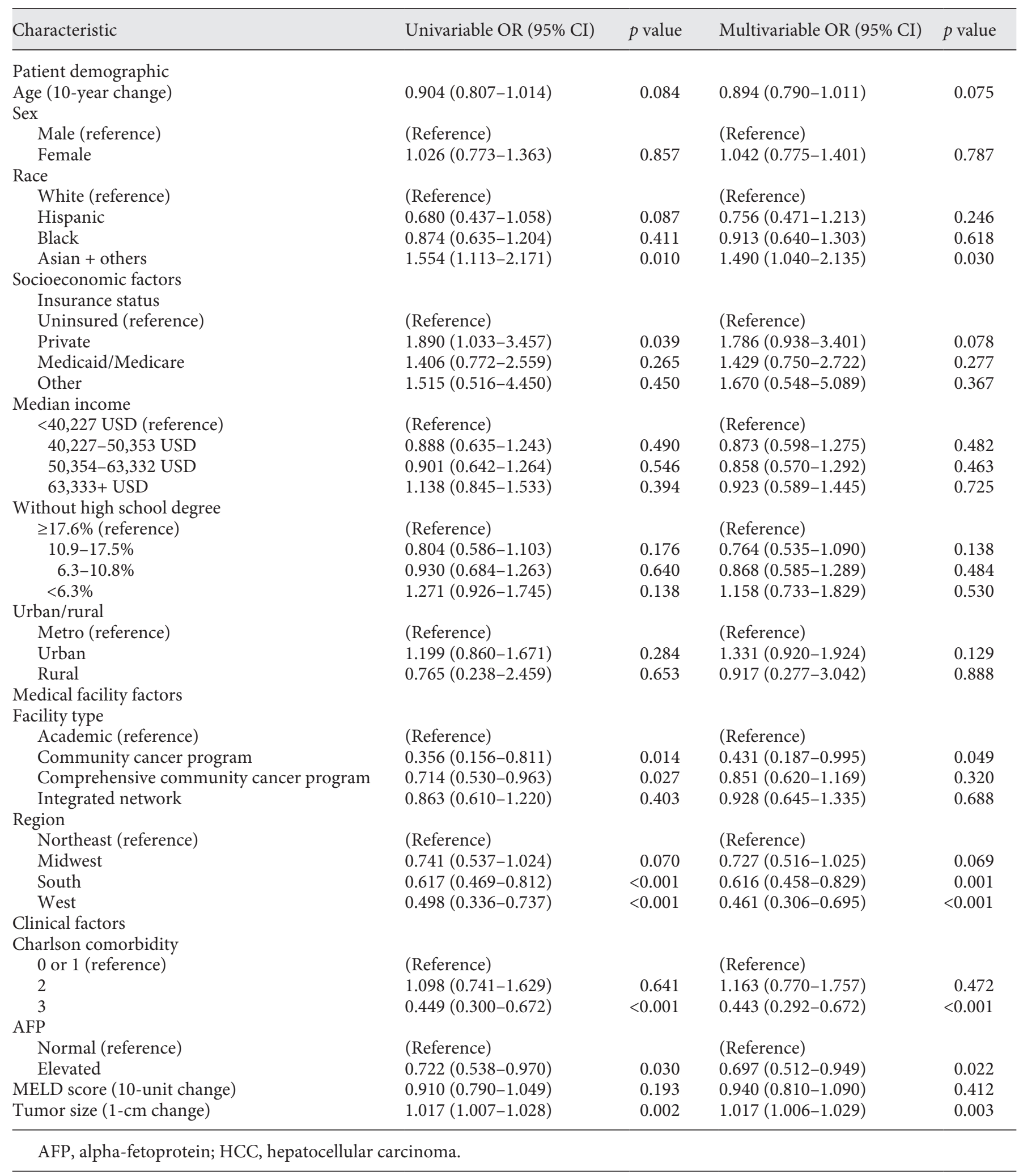


However, surgical resection was performed in less than $3 \%$ of patients, suggesting it is currently used in a highly selected subgroup of patients. Outside of clinical characteristics such as comorbidity index and AFP level, we noted significant geographic and center-level variation in the use of surgical resection for patients with vascular invasion.

Conventionally, patients with vascular invasion are categorized as BCLC stage $\mathrm{C}$ with advanced cancer [5]. Systemic treatment is recommended for the management of HCC associated with macrovascular invasion. Our current understanding of the efficacy of surgical interventions in advanced HCC is mainly from small retrospective studies from Asia and Europe. The results of our study are consistent with those of the other smaller studies done outside of the USA $[15,16,25,26]$. One of the early cases of liver resection for HCC for patients with portal vein invasion was reported in 1990 [27]. Ever since multiple small studies have reported the utilization of surgery in patients with vascular invasion although most studies lack comparison with the standard of care treatment [28-30]. Kokoda et al. performed a nationwide multicenter analysis in Japan and showed that liver resection is associated with improved survival in those with portal vein tumor thrombosis limited to the firstorder or peripheral branch with median survival time (1.77 years longer) compared to the nonsurgical group [15]. Subsequently, they also showed improvement in median survival time (2.89 years longer) in those with hepatic vein tumor invasion compared to the nonresection group [31,32]. Peng et al. [33] showed that hepatic resection improved patients' 5-year overall survival (11.1\%) compared to those who had TACE $(0.5 \%)$. Other studies in Asia and European countries have described survival benefits with aggressive surgical resection in several medical centers, mostly for vascular invasion restricted to the segmental branches or the right/ left portal/hepatic vein without invasion of the main portal vein $[18,34-36]$.

While there is no large-scale cohort study that investigated surgical resection for HCC with vascular invasion in the USA, one multicenter retrospective study compiled data from the University of Texas M. D. Anderson Cancer Center (Houston, TX, USA), Mayo Clinic (Rochester, MN, USA), Beaujon Hospital (Paris, France), Kyoto University Graduate School of Medicine (Kyoto, Japan), and Queen Mary Hospital (Hong Kong, China) and reported the clinical outcome of 102 patients with HCC and vascular invasion who underwent resection between 1984 and 1999. The author reported me-

Hepatocellular Carcinoma with Vascular Invasion dian survival was 11 months, and the severity of liver fibrosis was the only independent prognostic factor [18]. The study lacked a control group who received systemic treatment, and patients included in this study received surgical resection 2-3 decades ago. Postsurgical outcome of HCC has substantially improved for the past few decades [37]; thus, study finding may be less applicable in determining the outcome of surgical resection in a contemporary cohort of HCC patients.

We also noted that patients who had $\mathrm{R} 1$ or $\mathrm{R} 2$ resection had longer OS than patients who received systemic treatment. This raises questions on the therapeutic role of debulking surgery in advanced HCC with preserved liver function. Survival benefit of liver resection has been reported in advanced-stage solid organ cancer patients with hepatic metastasis $[38,39]$. Although the risk of perioperative hepatic decompensation should be carefully measured, the concept of surgical debulking followed by immuno-oncological management in highrisk patients warrants further investigation.

Finally, we reported that demography, facility type, and regions within the USA are associated with the receipt of surgical resection. First, we found that Asian race was associated with a higher likelihood of receiving surgical resection, likely attributed to a higher proportion of HBV-induced HCC in Asian patients with less hepatic dysfunction. Indeed, the proportion of patients with cirrhosis was lower in Asian versus non-Asian (68 vs. $82 \%, p<0.001$ ), while MELD score was lower in Asian versus non-Asian (10.5 vs. $11.2, p=0.048)$. Patients receiving cares at an academic cancer center was associated with the higher likelihood of receiving surgical resection, likely due to their expertise in the selection of surgical candidate, surgical skills, and postsurgical management.

This study has several limitations. This is a retrospective study, and some pertinent data were not available or were missing. For example, the Child-Pugh score and degree of portal hypertension were not available. Cirrhosis status was missing in more than three-quarters of patients and was not included in our logistic and Cox regression models. To address this concern, analyses were adjusted using MELD scores to reflect the severity of liver dysfunction. In addition, sensitivity analysis with stratification/propensity score matching of cirrhosis status confirmed the superiority of surgical resection. While the extent of vascular invasion is a key prognostic variable in HCC with macrovascular invasion, these data were also not available, and there is a possibility of residual confounding despite statistical adjustments. 
However, the survival of 21 months for those who received surgical resection in the current study is far better than a median survival time of 14.5 months for ChildPugh A HCC patients without vascular invasion or metastasis treated with sorafenib in the SHARP trial [13]. The NCDB may have potential issues regarding generalizability because it only covers patients presented at participating institutions, although it covers over $70 \%$ of all cancer cases in the USA. The database does not capture all longitudinal treatment and clinical courses, including post-treatment recurrence and cause of death. Of note, 107 patients (33\%) who underwent surgical resection had received systemic treatment before or after surgery, but their survival was not different from those who received surgical resection without systemic treatment $(p=0.33)$. Finally, patients included in this study were diagnosed in 2017 or before and presumably received sorafenib as the first line of systemic treatment although specific types of systemic therapy are not available in the NCDB. There have been several advances in both first- and second-line therapy since 2017, including the introduction of atezolizumab and bevacizumab, showing superior survival than sorafenib in the frontline setting [14, 40-46]. Therefore, the superiority of surgical management to more contemporary systemic treatment remains unclear and should be further investigated in future studies. The idea of surgical resection for resectable HCC with tumor thrombus is highly controversial, and randomized controlled trials are needed to guide treatment allocation. This question is particularly relevant in the era of immuno-oncological treatment modality showing highly promising efficacy in patients with advanced HCC.

In conclusion, our analysis of a large contemporary cohort of HCC patients showed that surgical resection could be one of the treatment options in highly selected patients with HCC and vascular invasion. This is the largest comparative effectiveness study of HCC patients with vascular invasion who underwent surgical resection in the USA, and we reported that surgical resection is currently used in a minority of patients, including notable variation by several patients and treating facilityrelated characteristics. These data highlight that randomized controlled trials should be considered to determine the role of surgical resection for resectable HCC patients with macrovascular invasion with good liver function.

\section{Statement of Ethics}

The study does not require Ethical Committee approval as the study analyzed a deidentified dataset obtained from the NCDB. Our research complies with the guidelines for human studies and was conducted ethically in accordance with the World Medical Association Declaration of Helsinki.

\section{Conflict of Interest Statement}

Dr. Yang provides a consulting service for Exact Sciences, Eisai and Gilead. Dr. Singal has been on advisory boards and served as a consultant for Genentech, Bayer, Eisai, BMS, Exelixis, AstraZeneca, and TARGET RWE. Dr. Roberts is an editorial board member of Liver Cancer. The other authors have no potential conflict of interest.

\section{Funding Sources}

Dr. Yang's research is supported by the American College of Gastroenterology Junior Faculty Development Award, Department of Defense Peer Reviewed Cancer Research Program Career Development Award, Cedars-Sinai Clinical Scholar Award, and Huiying Foundation. Dr. Singal's research is funded by the National Institutes of Health R01 MD12565.

\section{Author Contributions}

Substantial contributions to conception and design (Drs. Ahn, Kosari, Todo, Kim, Noureddin, Kuo, Walid, Sundaram, Lu, Roberts, Singal, Heimbach, Agopian, Nissen, and Yang); acquisition of data, and/or analysis and interpretation of data (Dr. Govalan, Ms. Lauzon, and Mr. Luu); drafting the manuscript (Dr. Govalan and Ms. Lauzon) or revising it critically for important intellectual content (Mr. Luu, Drs. Ahn, Kosari, Todo, Kim, Noureddin, Kuo, Walid, Sundaram, Lu, Roberts, Singal, Heimbach, Agopian, Nissen, and Yang); and final approval of the version to be published (Dr. Govalan, Ms. Lauzon, Mr. Luu, Drs. Ahn, Kosari, Todo, Kim, Noureddin, Kuo, Walid, Sundaram, Lu, Roberts, Singal, Heimbach, Agopian, Nissen, and Yang MD, MS).

\section{Role of the Funder/Sponsor}

The American College of Gastroenterology, Department of Defense, and Cedars-Sinai Medical Center, and Huiying Foundation, and NIH had no role in the collection of data; the design and conduct of the study; management, analysis, and interpretation of the data; preparation, review, or approval of the manuscript; and decision to submit the manuscript for publication. 


\section{References}

1 Bray F, Ferlay J, Soerjomataram I, Siegel RL, Torre LA, Jemal A. Global cancer statistics 2018: GLOBOCAN estimates of incidence and mortality worldwide for 36 cancers in 185 countries. CA Cancer J Clin. 2018 Nov;68(6): 394-424.

2 Yang JD, Hainaut P, Gores GJ, Amadou A, Plymoth A, Roberts LR. A global view of hepatocellular carcinoma: trends, risk, prevention and management. Nat Rev Gastroenterol Hepatol. 2019 Oct;16(10):589-604.

3 Kulik L, El-Serag HB. Epidemiology and management of hepatocellular carcinoma. Gastroenterology. 2019 Jan;156(2):477-e1.

4 Marrero JA, Kudo M, Bronowicki JP. The challenge of prognosis and staging for hepatocellular carcinoma. Oncologist. 2010; 15(Suppl 4):23-33.

5 Llovet JM, Brú C, Bruix J. Prognosis of hepatocellular carcinoma: the BCLC staging classification. Semin Liver Dis. 1999;19(3):329_ 38.

6 Yau T, Tang VY, Yao TJ, Fan ST, Lo CM, Poon RT. Development of Hong Kong liver cancer staging system with treatment stratification for patients with hepatocellular carcinoma. Gastroenterology. 2014 Jun; 146(7): 1691-e3.

7 Forner A, Reig M, Bruix J. Hepatocellular carcinoma. Lancet. 2018 Mar 31;391(10127): 1301-14.

8 Marrero JA, Kulik LM, Sirlin CB, Zhu AX, Finn RS, Abecassis MM, et al. Diagnosis, staging, and management of hepatocellular carcinoma: 2018 practice guidance by the American Association for the Study of Liver Diseases. Hepatology. 2018 Aug;68(2):723-50.

9 Han KH, Kudo M, Ye SL, Choi JY, Poon RT, Seong J, et al. Asian consensus workshop report: expert consensus guideline for the management of intermediate and advanced hepatocellular carcinoma in Asia. Oncology. 2011; 81(Suppl 1):158-64.

10 Kanwal F, Singal AG. Surveillance for hepatocellular carcinoma: current best practice and future direction. Gastroenterology. 2019 Jul; 157(1):54-64

11 Llovet JM, Ricci S, Mazzaferro V, Hilgard P, Gane E, Blanc JF, et al. Sorafenib in advanced hepatocellular carcinoma. N Engl J Med. 2008 Jul 24;359(4):378-90.

12 Cheng AL, Kang YK, Chen Z, Tsao CJ, Qin S, Kim JS, et al. Efficacy and safety of sorafenib in patients in the Asia-Pacific region with advanced hepatocellular carcinoma: a phase III randomised, double-blind, placebo-controlled trial. Lancet Oncol. 2009 Jan;10(1): 25-34.

13 Bruix J, Raoul JL, Sherman M, Mazzaferro V, Bolondi L, Craxi A, et al. Efficacy and safety of sorafenib in patients with advanced hepatocellular carcinoma: subanalyses of a phase III trial. J Hepatol. 2012 Oct;57(4):821-9.
14 Kudo M, Finn RS, Qin S, Han KH, Ikeda K, Piscaglia $F$, et al. Lenvatinib versus sorafenib in first-line treatment of patients with unresectable hepatocellular carcinoma: a randomised phase 3 non-inferiority trial. Lancet. 2018 Mar 24;391(10126):1163-73.

15 Kokudo T, Hasegawa K, Matsuyama Y, Takayama T, Izumi N, Kadoya M, et al. Survival benefit of liver resection for hepatocellular carcinoma associated with portal vein invasion. J Hepatol. 2016 Nov;65(5):938-43.

16 Minagawa M, Makuuchi M, Takayama T, Ohtomo K. Selection criteria for hepatectomy in patients with hepatocellular carcinoma and portal vein tumor thrombus. Ann Surg. 2001 Mar;233(3):379-84.

17 Ikai I, Yamamoto Y, Yamamoto N, Terajima H, Hatano E, Shimahara Y, et al. Results of hepatic resection for hepatocellular carcinoma invading major portal and/or hepatic veins. Surg Oncol Clin N Am. 2003 Jan;12(1): 65-75.

18 Pawlik TM, Poon RT, Abdalla EK, Ikai I, Nagorney DM, Belghiti J, et al. Hepatectomy for hepatocellular carcinoma with major portal or hepatic vein invasion: results of a multicenter study. Surgery. 2005 Apr;137(4):40310.

19 Torzilli G, Donadon M, Marconi M, Palmisano A, Del Fabbro D, Spinelli A, et al. Hepatectomy for stage $\mathrm{B}$ and stage $\mathrm{C}$ hepatocellular carcinoma in the Barcelona Clinic Liver Cancer classification: results of a prospective analysis. Arch Surg. 2008 Nov; 143(11):1082-90.

20 Roayaie S, Jibara G, Tabrizian P, Park JW, Yang J, Yan L, et al. The role of hepatic resection in the treatment of hepatocellular cancer. Hepatology. 2015 Aug;62(2):440-51.

21 White IR, Royston P, Wood AM. Multiple imputation using chained equations: Issues and guidance for practice. Stat Med. $2011 \mathrm{Feb}$ 20;30(4):377-99.

22 Austin PC. An introduction to propensity score methods for reducing the effects of confounding in observational studies. Multivariate Behav Res. 2011 May;46(3):399-424.

23 Austin PC. A comparison of 12 algorithms for matching on the propensity score. Stat Med. 2014 Mar 15;33(6):1057-69.

24 Grambsch PM, Therneau TM. Proportional hazards tests and diagnostics based on weighted residuals. Biometrika. 1994;81(3): 515-26.

25 Ng KK, Vauthey JN, Pawlik TM, Lauwers GY, Regimbeau JM, Belghiti J, et al. Is hepatic resection for large or multinodular hepatocellular carcinoma justified? Results from a multi-institutional database. Ann Surg Oncol. 2005 May;12(5):364-73.

26 Wilson GC, Geller DA. Evolving surgical options for hepatocellular carcinoma. Surg Oncol Clin N Am. 2019 Oct;28(4):645-61.
27 Kumada K, Ozawa K, Okamoto R, Takayasu T, Yamaguchi M, Yamamoto Y, et al. Hepatic resection for advanced hepatocellular carcinoma with removal of portal vein tumor thrombi. Surgery. 1990 Nov;108(5):821-7.

28 Inoue $\mathrm{Y}$, Hasegawa $\mathrm{K}$, Ishizawa $\mathrm{T}$, Aoki $\mathrm{T}$, Sano K, Beck Y, et al. Is there any difference in survival according to the portal tumor thrombectomy method in patients with hepatocellular carcinoma? Surgery. 2009 Jan; 145(1):9-19.

29 Irtan S, Chopin-Laly X, Ronot M, Faivre S, Paradis V, Belghiti J. Complete regression of locally advanced hepatocellular carcinoma induced by sorafenib allowing curative resection. Liver Int. 2011 May;31(5):740-3.

30 Shaohua L, Qiaoxuan W, Peng S, Qing L, Zhongyuan Y, Ming S, et al. Surgical strategy for hepatocellular carcinoma patients with portal/hepatic vein tumor thrombosis. PLoS One. 2015;10(6):e0130021

31 Kokudo T, Hasegawa K, Yamamoto S, Shindoh J, Takemura N, Aoki T, et al. Surgical treatment of hepatocellular carcinoma associated with hepatic vein tumor thrombosis. J Hepatol. 2014 Sept;61(3):583-8.

32 Kokudo T, Hasegawa K, Matsuyama Y, Takayama T, Izumi N, Kadoya M, et al. Liver resection for hepatocellular carcinoma associated with hepatic vein invasion: a Japanese nationwide survey. Hepatology. 2017 Aug; 66(2):510-7.

33 Peng ZW, Guo RP, Zhang YJ, Lin XJ, Chen MS, Lau WY. Hepatic resection versus transcatheter arterial chemoembolization for the treatment of hepatocellular carcinoma with portal vein tumor thrombus. Cancer. 2012 Oct 1;118(19):4725-36.

34 Pesi B, Ferrero A, Grazi GL, Cescon M, Russolillo $\mathrm{N}$, Leo $\mathrm{F}$, et al. Liver resection with thrombectomy as a treatment of hepatocellular carcinoma with major vascular invasion: results from a retrospective multicentric study. Am J Surg. 2015 Jul;210(1):35-44.

35 Zhang XP, Wang K, Chen ZH, Cheng SQ. Hepatocellular carcinoma with hepatic vein invasion should not be considered a contraindication for liver resection. Hepatology. 2018 Feb;67(2):804-5.

36 Zhang ZY, Dong KS, Zhang EL, Zhang LW, Chen XP, Dong HH. Resection might be a meaningful choice for hepatocellular carcinoma with portal vein thrombosis: a systematic review and meta-analysis. Medicine. 2019 Dec;98(50):e18362.

37 Allaire M, Goumard C, Lim C, Le Cleach A, Wagner M, Scatton O. New frontiers in liver resection for hepatocellular carcinoma. JHEP Reports. 2020 Aug;2(4):100134.

38 Rees M, Tekkis PP, Welsh FK, O’Rourke T, John TG. Evaluation of long-term survival after hepatic resection for metastatic colorectal cancer: a multifactorial model of 929 patients. Ann Surg. 2008 Jan;247(1):125-35. 
39 Rahnemai-Azar AA, Selby LV, Lustberg MB, Pawlik TM. Surgical management of breast cancer liver metastasis. Surg Oncol Clin N Am. 2021 Jan;30(1):27-37.

40 Bruix J, Qin S, Merle P, Granito A, Huang YH, Bodoky G, et al. Regorafenib for patients with hepatocellular carcinoma who progressed on sorafenib treatment (RESORCE): a randomised, double-blind, placebo-controlled, phase 3 trial. Lancet. 2017 Jan 7;389(10064): 56-66.

41 El-Khoueiry AB, Sangro B, Yau T, Crocenzi TS, Kudo M, Hsu C, et al. Nivolumab in patients with advanced hepatocellular carcinoma (CheckMate 040): an open-label, noncomparative, phase $1 / 2$ dose escalation and expansion trial. Lancet. 2017 Jun 24; 389(10088):2492-502.

42 Abou-Alfa GK, Meyer T, Cheng AL, ElKhoueiry AB, Rimassa L, Ryoo BY, et al. Cabozantinib in patients with advanced and progressing hepatocellular carcinoma. $\mathrm{N}$ Engl J Med. 2018 Jul 5;379(1):54-63.

43 Finn RS, Zhu AX, Farah W, Almasri J, Zaiem F, Prokop LJ, et al. Therapies for advanced stage hepatocellular carcinoma with macrovascular invasion or metastatic disease: a systematic review and meta-analysis. Hepatology. 2018 Jan;67(1):422-35.

44 Finn RS, Qin S, Ikeda M, Galle PR, Ducreux M, Kim TY, et al. Atezolizumab plus Bevacizumab in unresectable hepatocellular carci- noma. N Engl J Med. 2020 May 14;382(20): 1894-905.

45 Finn RS, Ryoo BY, Merle P, Kudo M, Bouattour M, Lim HY, et al. Pembrolizumab as second-line therapy in patients with advanced hepatocellular carcinoma in KEYNOTE-240: a randomized, double-blind, phase III trial. J Clin Oncol. 2020 Jan 20; 38(3): 193-202.

46 Yau T, Kang YK, Kim TY, El-Khoueiry AB, Santoro A, Sangro B, et al. Efficacy and safety of nivolumab plus ipilimumab in patients with advanced hepatocellular carcinoma previously treated with sorafenib: the checkmate 040 randomized clinical trial. JAMA Oncol. 2020 Oct 1;6(11):e204564. 